elevato nell'intervallo di tempo prossimo all'esordio clinico, e tendono a decrescere successivamente.

Il titolo IF correla inversamente sia con l'AI che con la distanza del prelievo dall'esordio clinico $(\mathrm{P}<0.001)$.

$\mathrm{Al}$ contrario, il titolo NAb correla positivamente con l'AI e con la distanza dall'esordio clinico $(\mathrm{P}<0.001)$.

\title{
Conclusioni:
}

L'AI ed il titolo NAb contro il SARS-CoV aumentano progressivamente con la distanza dall'esordio clinico, mentre il titolo IF è meno stabile nel tempo.

Pertanto la NAb è più adatta della IF per diagnosticare la pregressa infezione da SARS-CoV.

Inoltre la combinazione di NAb ed AI può fornire informazioni utili per una datazione approssimativa dell'epoca dell'infezione, ed è pertanto applicabile anche ad indagini sieroepidemiologiche sulla circolazione del SARS-CoV nella popolazione umana.

\section{CONFRONTO TRA IMMUNOFLUORESCENZA, NEUTRALIZZAZIONE E INDICE DI AVIDITA' NELLA RILEVAZIONE DELLA RISPOSTA ANTICORPALE ANTI-SARS-CoV}

\author{
Di Caro A. ', Castilletti C.', Chiappini R.', Travaglini D.', \\ Girardi E. ${ }^{2}$, Chan P. ${ }^{3}$, Capobianchi M.R.' \\ 'Laboratorio di Virologia, ²Dipartimento di Epidemiologia, Istituto \\ Nazionale per le Malattie Infettive INMI "L. Spallanzani", Roma, \\ ${ }^{3}$ Chinese University of Hong Kong, Prince of Wales Hospital. \\ Hong Kong, China
}

\section{Introduzione:}

Sebbene l'epidemia di SARS del 2002-2003 non si sia ripetuta, il succedersi di piccoli focolai, spesso dovuti ad incidenti di laboratorio, e la possibilità che il SARS-CoV possa continuare a circolare nei reservoir naturali, rende opportuna la definizione di metodi diagnostici per mantenere la vigilanza, ed approfondire la epidemiologia e la storia naturale. Abbiamo valutato l'andamento della risposta anticorpale verso SARS-CoV in un gruppo di pazienti cinesi, utilizzando l'immunofluorescenza (IF), la sieroneutralizzazione $(\mathrm{NAb})$ e l'indice di avidità delle $\mathrm{IgG}(\mathrm{AI})$.

Metodi:

Sono studiati 57 pazienti coinvolti nell'epidemia del $2003 \mathrm{ad}$ Hong Kong (27 maschi e 30 femmine, età media 41.25 anni). I campioni consecutivi saggiati sono stati: due per 23 pazienti (intervallo 18-253 giorni, media 123), tre per 2 pazienti, uno per i restanti 31 pazienti.

La determinazione del titolo IF e dell'AI è stata eseguita con metodi precedentemente descritti.

Il test di neutralizzazione è stato eseguito utilizzando il ceppo TOR2 di SARS-CoV in laboratorio di biosicurezza di livello 3. L'analisi dei dati è stata eseguita utilizzando il software SPP.

Risultati:

Gli anticorpi rilevabili in IF hanno un titolo mediamente più 